CERN LIBRARIES, GENEVA

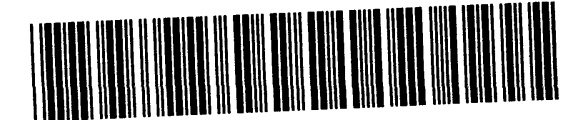

CERN-TH. 4619

CM-P00062398

\title{
LUND MODEL AND AN OUTSIDE-INSIDE ASPECT OF THE INSIDE-OUTSIDE CASCADE
}

\author{
A. Białas \\ CERN - Geneva \\ and \\ Institute of Physics, Jagellonian University, Cracow
}

\author{
M. Gyulassy \\ CERN - Geneva \\ and \\ Nuclear Science Division \\ Lawrence Berkeley Laboratory, Berkeley CA 94720
}

\section{A B S T R A C T}

The formation zone of high-energy hadrons is discussed based on the Lund model of hadronization. The ambiguity in the concept of formation length for composite particles is pointed out. Two different formation lengths are defined and their distributions calculated. The results are compared with the data on fragmentation of $30 \mathrm{GeV}$ pions into protons and antiprotons. The data indicate the possibility of an outside-inside cascade picture of hadronization in the projectile fragmentation region. 


\section{INTRODUCTION}

One of the primary motivations for studying hadron-nucleus collisions is to learn about the space-time development of hadronization. The present understanding of this space-time development is that high-energy secondary hadrons tend to be produced after low-energy ones [1-3]. This feature, referred to as the 'insideoutside' cascade [4], follows from very general arguments based only on time dilation and the uncertainty principle [1-9]. In its simplest form, if $\tau_{0}$ denotes the proper formation time of a hadron of transverse mass $m_{\perp}$, then the formation time $t$ or length $z$ in a frame where that particle has energy $E$ is dilated as

$$
t \approx z \approx \tau_{0} E / m_{\perp}
$$

A strict definition of formation time can of course apply only to the production of structureless particles for which the uncertainty principle provides a natural scale $\tau_{0} \sim \hbar / m_{\perp}$. For composite particles (such as hadrons) the very notion of formation time is ambiguous because, for example, different constituents of a hadron can be produced at different times. It is then an open and model-dependent question of which time is relevant for nuclear attenuation. The idea that the formation zone may be different -- and in fact much shorter than given by eq. (1.1) -- was first expressed in ref. [10] and then applied to the analysis of several processes [11, 12]. In the present paper we study this problem in the context of the Lund model of hadronization [13]. Our approach follows that started by Chmaj [14] who proposed a specific definition of the formation time of a high-energy hadron and calculated its average value predicted by the Lund model. Our analysis extends the work of ref.

[14] in three directions:

i) We discuss in detail the above-mentioned ambiguity in the concept of formation time associated with the composite character of hadrons: although the asymptotic hadronic state becomes well-defined only at large distances from the point of interaction in accord with eq. (1.1), some of the hadronic constituents are formed rather early and may interact in the nucleus before the final hadron is formed.

ii) The distribution of these different formation lengths is derived, and employed in a calculation of nuclear attenuation in the projectile fragmentation region. 
iii) These results are compared with the data on fragmentation of $30 \mathrm{GeV}$ pions into $p$ and $\vec{p}[15]$. These data indicate that the formation length determining the absorption of a hadron in nuclear matter is smaller than the 'yo-yo length' of the Lund model as defined in section 3, and is consistent with the point at which the first constituent of this hadron appears. This 'constituent length' decreases (on the average) with increasing energy of the final hadron in the fragmentation region and thus corresponds to an outside-inside picture of hadronization.

While the picture we obtain is model-dependent, it leads to further specific consequences which can be tested experimentally.

The remainder of this paper is organized as follows. In section 2 we discuss the relation between nuclear attenuation and the formation length. In section 3 the ambiguity of the formation length in the Lund model is discussed and two extreme scales, called the 'yo-yo length' $1_{y}$ and 'constituent length' $1_{c}$ ' are defined. In section 4 the distribution functions of $l_{C}$ and $l_{y}$ are derived. In section 5 we consider possible extensions of the Lund model to hadron-nucleus collisions and compare results with experimental data. Our conclusions are given in section 6 .

\section{FORMATION LENGTH AND A-DEPENDENCE OF PROJECTILE FRAGMENTATION}

The exact value of the formation length is reflected in many aspects of interactions in nuclei. In this paper we discuss how finite formation lengths influence nuclear attenuation in the projectile fragmentation region, extending the work of ref. [16].

We consider an inclusive process

$$
h_{i}+A \rightarrow h_{f}+X
$$

where the fragment $h_{f}$ takes a large fraction $x$ of the energy of the projectile $h_{i}$. For a non-vanishing formation length the mechanism for this process is shown in fig. 1; the projectile $h_{i}$ interacts inelastically in the target and a new intermediate state $h^{*}$ is created. This intermediate state later fragments into the final hadron $h_{f}$. The distance from the point of interaction to the point of fragmentation is the formation length 1 of the hadron $h_{f}$. 
The difference between an elementary and a nuclear target is that in the nucleus the projectile $h_{i}$, the intermediate state $h^{*}$, and the final hadron $h_{f}$ have to pass through nuclear matter, and hence are subject to attenuation due to inelastic rescattering. Consequently, the cross-section for the process (2.1) can be written as

$$
d \sigma\left(h_{i} \rightarrow h_{f} ; A\right)=d \sigma_{0}+d \sigma_{1}+d \sigma_{2}+\cdots
$$

where $d \sigma_{k}=d \sigma_{k}\left(h_{i} \rightarrow h_{f} ; A\right)$ is the contribution from the process with $k$ inelastic rescatterings inside the nucleus. Thus, to get a complete description of the reaction (2.1) on nuclear targets it is necessary to calculate all the terms of the series (2.2). We note, however, that the projectile fragmentation region, i.e. the kinematic range where the fragment $h_{f}$ takes a large fraction $x$ of the energy of the projectile, is likely to be dominated by the first term in eq. (2.2), i.e. by the contribution with no rescattering. This conclusion follows from the observation that any inelastic rescattering reduces the energy of the particle and, consequently, the terms $\sigma_{1}, \sigma_{2}, \ldots$ contribute to smaller $x$ values than $\sigma_{0}$. The crosssections for most of the processes (2.1) are known to fall rapidly when $x \rightarrow 1$. This implies that even a relatively small shift in $x$ strongly reduces the cross-section at a given $x$.

To summarize, in the projectile fragmentation region we expect

$$
d \sigma\left(h_{i} \rightarrow h_{f} ; A\right) \approx d \sigma_{0}\left(h_{i} \rightarrow h_{f} ; A\right)=A P_{0} \operatorname{d\sigma }\left(h_{i} \rightarrow h_{f} j 1\right)
$$

where $P_{0}$ is the probability that no rescattering of $h_{i}, h^{*}$, or $h_{f}$ takes place in nuclear matter. Consequently, the ratio

$$
R_{A} \equiv \frac{d \sigma\left(h_{i} \rightarrow h_{f} j A\right)}{A d \sigma\left(h_{i} \rightarrow h_{f} ; 1\right)} \approx P_{0}
$$

measures the probability of no rescattering in the nucleus $A$.

To calculate $P_{0}$ at fixed impact parameter $b$, we note that if the first interaction of $h_{1}$ occurs at depth $z$ and the final fragment $h_{f}$ forms out of the intermediate state $h^{*}$ at depth $z^{\prime}$, then the probability of no rescattering of $h_{i}$, $h^{*}$, or $h_{f}$ is

$$
W_{0}\left(z, z^{\prime}\right)=\left(1-W_{i}(z)-W^{*}\left(z, z^{\prime}\right)-W_{f}\left(z^{\prime}\right)\right)^{A-1}
$$


where

$$
\begin{aligned}
W_{i}(z) & =\int_{-\infty}^{z} d z \sigma_{i} \rho(z, b) \\
W^{*}\left(z, z^{\prime}\right) & =\int_{z}^{z^{\prime}} d z \sigma^{*} \rho(z, b) \\
W_{f}\left(z^{\prime}\right) & =\int_{z^{\prime}}^{\infty} d z \quad \sigma_{f} \rho(z, b)
\end{aligned}
$$

Here $e(\vec{x})$ is the normalized nuclear density. The cross-sections $\sigma_{i}, \sigma^{*}, \sigma_{f}$ correspond to the inelastic non-diffractive cross-sections of $h_{i}, h^{*}, h_{f}$, respectively. Equation (2.5) has the simple geometrical interpretation shown in fig. 1 . Note that $w_{i}$ is the fraction of the total nuclear volume swept out by $h_{i} ; W^{*}$ is the fraction swept out by $h^{\star}$, and $w_{f}$ is the fraction swept out by $h_{f}$. The condition for no rescattering just corresponds to demanding that none of the A - 1 target nucleons happens to be inside the volume swept out by the traversing $h_{i} \rightarrow h^{*} \rightarrow h_{f}$ system. of course, one of the target nucleons must interact with $h_{i}$. The probability that $h_{i}$ will interact between $z$ and $z+d z$ is $A_{i} \rho(z, b) d z$.

Now let $D(x, \Delta z) d x d z$ denote the number of $h_{f}$ forming out of $h^{*}$ at a distance $\Delta z$ from the interaction point of $h_{i}$, carrying the fraction $x=(E+p) /\left(E_{i}+p_{i}\right)$ of the initial light-cone momentum. The distribution $d N / d x$ of $h_{f}$ in momentum space is given by

$$
\frac{d N}{d x}=\int_{0}^{\infty} d z D(x, z) \equiv D(x)
$$

The specific form of $D(x, z)$ in the context of the Lund model is derived in section 4 . The inclusive cross-section for $h_{i}+A+h_{f}$ is then given by

$$
\begin{gathered}
\frac{d \sigma_{0}}{d x}\left(h_{i} \rightarrow h_{f} ; A\right)= \\
A \int d^{2} b \int_{-\infty}^{\infty} d z \sigma_{i} \rho(z, b) \int_{z}^{\infty} d z^{\prime} D\left(x, z^{\prime}-z\right) W_{0}\left(z, z^{\prime}\right)
\end{gathered}
$$

For $A=1, Q(x)=\delta^{3}(x)$, and eq. (2.8) reduces to

$$
\frac{d \sigma}{d x}\left(h_{i} \rightarrow h_{f} j 1\right)=\sigma_{i} \int_{0}^{\infty} d z D(x, z)=\sigma_{i} \cdot \frac{d N}{d x}
$$

The attenuation ratio in the approximation $(2.4)$ is then given by 


$$
\begin{aligned}
& R_{A} \approx P_{0}= \\
& D^{-1}(x) \int d^{2} b \int_{-\infty}^{\infty} d z \rho(z, b) \int_{z}^{\infty} d z^{\prime} D\left(x, z^{\prime}-z\right)\left(1-W_{i}-W^{*}-W_{f}\right)^{A-1}
\end{aligned}
$$

We note that in ref. [16] a different formula for $P_{0}$ was employed. It was assumed that the cross-section of the intermediate hadronic state interpolates smoothly between $\sigma^{*}$ and $\sigma_{f}$. This is only an approximation whereas eq. (2.10) gives an exact solution to this problem.

We note two important limits of $(2.10)$ in which $P_{0}$ is determined solely by geometry: (i) $\sigma^{*}=\sigma_{f}=0$ and (ii) $\sigma^{*}=\sigma_{f}=\sigma_{i}$. In both cases $w_{0}\left(z, z^{\prime}\right)$ ceases to depend on the formation depth $z^{\prime}$ and $D(x, \Delta z)$ drops out of (2.10). In the first case

$$
\begin{aligned}
P_{0} & =\int d^{2} b \int_{-\infty}^{\infty} d z \rho(z, b)\left(1-\int_{-\infty}^{z} d z^{\prime} \sigma_{i} \rho\left(z^{\prime}, b\right)\right)^{A-1} \\
& =\left(A \sigma_{i}\right)^{-1} \int d^{2} b\left\{1-(1-N(b))^{A}\right\} \\
& =\sigma_{i A} / A \sigma_{i} \equiv 1 / \nu_{i A}
\end{aligned}
$$

where $\mathrm{AN}(\mathrm{b})=\mathrm{A} \int \mathrm{dzo} \mathrm{O}_{\mathrm{i}} \varrho(\mathrm{z}, \mathrm{b})$ is the average number of inelastic interactions at impact parameter $b, \sigma_{i A}$ is just the total $h_{i}+A$ inelastic cross-section, and $v_{i A}$ is the average number of inelastic collisions integrated over impact parameters.

In the second limit,

$$
P_{0}=\int \frac{d^{2} b}{\sigma_{i}} N(b)(1-N(b))^{A-1}=p_{i A}(1) / \nu_{i A}
$$

where $p_{i A}(1)$ is the Glauber probability that $h_{i}$ suffers only one inelastic scatbering in $A$.

From eq. (2.10) we therefore see that measurements of nuclear attenuation allow us to obtain information on the formation-length distribution $D(x, \Delta z)$ only if $\sigma^{*}$ and $\sigma_{f}$ are not close to the above limits. However, because of the convolution of effects

5 
in eq. (2.8), it is necessary to consider several sets of data to disentangle the sought-after information on $D(x, z)$. Fortunately, there are several experimental ways to attack this problem through variations of $h_{i}, h_{f}, A_{,} x_{f}$, and $E_{i}$. A specific example of such an analysis is presented in section 5 .

\section{FORMATION LENGTHS IN THE LUND MODEL}

The space-time development of hadronization in the Lund model is illustrated in fig. 2. It is a two-step process: first q̄ं pairs are created at points $p_{i}$ neutralizing the colour fields between them, and then neighbouring q $\bar{q}$ pairs join at points $H_{i}$ to form colour singlet 'yo-yo' states that represent hadrons. While the $H_{i}$ represent natural candidates for the formation points of the hadrons, as suggested by the authors of the Lund model $[13,14]$, it is possible that nuclear attenuation of the constituents of the hadrons may begin at an earlier point.

Consider, in particular, the leading hadron whose constituents first intersect at point $\mathrm{H}_{1}$ in fig. 2. We see that both constituents of that hadron were already present at the earlier point $P_{1}$. Between $P_{1}$ and $H_{1}$ the colour neutral 'yo-yo' pair that evolves in the leading hadron is difficult to distinguish from the asymptotic 'yo-yo' state beyond $\mathrm{H}_{1}$. It is thus possible that nuclear attenuation begins somewhere between $P_{1}$ and $H_{1}$, and perhaps as early as $P_{1}$. However, the 'pre-hadron' propagating beyond $P_{1}$ may not have the same cross-section as the asymptotic 'yo-yo' after $H_{1}$. For example, it may take some time for the current quark formed at $P_{1}$ to dress itself and behave as a constituent quark.

To study the consequence of this ambiguity in the definition of formation time, we will consider the two natural extremes of defining the formation point of the $n^{\text {th }}$-rank hadron as (i) $H_{n}$ or (ii) $P_{n}$. We refer to the $z$ coordinate of $H_{n}$ as the yo-yo formation length $l_{y}$ and to the $z$ coordinate of $P_{n}$ as the constituent formation length $1_{c}$.

In the Lund model $l_{y}$ and $l_{c}$ are related to each other in a simple way, independent of the rank of the hadron. The constituent formed with zero energy at $1_{c}$ accelerates to the right under an assumed-constant string tension $k$ ^ $1 \mathrm{GeV} / \mathrm{fm}$. By the time it reaches the point $H_{n}$ its light-cone momentum is $E+p=2 k\left(1_{y}-1_{c}\right)$. The other constituent of the $n^{\text {th }}$ rank 'yo-yo' formed at $P_{n-1}$ ' on the other hand, accelerates only to the left and its $E+p$ remains zero at the intersecting point $H_{n}$. 
More quantitatively, if $z_{n}=t_{n} \pm z_{n}$ denote the light-cone coordinates of the $n^{\text {th }}$ production point $P_{n}$, then the light-cone momenta $p_{n}^{ \pm}=E_{n} \pm p_{z n}$ of the $n^{\text {th }}$-rank hadron are fixed by [13]

$$
\begin{aligned}
& p_{n}^{+}=k\left(z_{n-1}^{+}-z_{n}^{+}\right) \\
& p_{n}^{-}=k\left(z_{n}^{-}-z_{n-1}^{-}\right)
\end{aligned}
$$

in terms of the string tension $k$. Since the transverse mass squared of the $n^{\text {th }}$-rank hadron is just $m_{n}^{2}=p_{n}^{+} p_{n}^{-}$, eq. (3.1) implies that for $p_{n}^{+} \gg m_{n}$, hadronization occurs very near the light-cone with

$$
z_{n}^{-}=k^{-1} \sum_{i=1}^{n} m_{i}^{2} / p_{i}^{+} \ll 1 f m
$$

From Fig. 2, it is obvious that $z_{0}^{-}=0$, while

$$
z_{0}^{+}=\left(E_{i}+p_{i}\right) / K \equiv 2 L
$$

is the distance at which the leading quark turns around. We refer to $L$ as the total length of the Lund string.

In terms of $z \frac{t}{n}$, the constituent formation length of the $n^{\text {th }}$-rank hadron at point $P_{n}$ is

$$
\ell_{c}=\frac{1}{2}\left(z_{n}^{+}-z_{n}^{-}\right)
$$

while the 'yo-yo' formation length at point $H_{n}$ is

$$
\ell_{y}=\frac{1}{2}\left(z_{n-1}^{+}-z_{n}^{-}\right)
$$

Therefore, using eq. (3.1),

$$
l_{y}-l_{c}=\frac{1}{2}\left(z_{n-1}^{+}-z_{n}^{+}\right)=x L
$$

where $x=p_{n}^{+} /\left(E_{0}+p_{0}\right)$ is the light-cone momentum fraction carried by the $n^{\text {th }}$-rank hadron and $L$ is given by eq. (3.3). For a fixed $x$, note that eq. (3.6) holds independently of the rank of the hadron.

Since $0 \leq x \leq 1$, we furthermore see that

$$
\begin{aligned}
& 0 \leq l_{c} \leq(1-x) L \\
& x L \leq l_{y} \leq L
\end{aligned}
$$


Therefore, near the kinematic limit $x \rightarrow 1,1_{y} \rightarrow L$ but the constituent formation length $1_{\mathrm{C}} \rightarrow 0$ !

From eq. (3.6) it is clear that the ambiguity in the definition of the formation length of a hadron becomes most acute for $x \rightarrow 1$. In that case, there is essentially only one very long 'yo-yo' whose constituents have formed very early after the interaction point.

The possible relevance of the constituent length, $1_{c}$, to nuclear attenuation is what we refer to as the outside-inside aspect of hadronization. of course for fixed $x$ all length scales $l_{C^{\prime}} l_{y}$, and $L$ increase linearly with increasing incident energy as expected from the general arguments leading to the inside-outside picture in eq. (1.1). However, what we see is that, within the context of the Lund model, the proportionality constant depends not only on the string tension $k$ but also on $x$ of the final fragment, as already noted in refs. [10], [11] and [14]. This peculiar twist of the inside-outside picture of hadronization arises in the Lund model because

i) hadrons are composite objects,

ii) their constituents are formed over a long time interval $x L$,

iii) some of these constituents could interact in nuclear matter long before they are all assembled to form the well-defined asymptotic hadron.

However, it is important to emphasize that the outside-inside picture of high-x particle formation is not specific to the Lund model. The original LandauPomeranchuk analysis of bremsstrahlung also reveals this effect. Consider the process where an incident particle with $\left(\mathrm{p}^{+}, \overrightarrow{\mathrm{p}}_{\perp}, \mathrm{p}^{-}\right)=\left(\mathrm{P}_{0}^{+}, 0, \mathrm{~m}^{2} / \mathrm{P}_{0}^{+}\right)$radiates, after a perturbation $\Delta \mathrm{p}^{-}$, a particle with $\left(\mathrm{xP}_{0}^{+}, \overrightarrow{\mathrm{p}}_{\perp}, \mathrm{m}_{\perp}^{2} / \mathrm{xP}_{0}^{+}\right)$, leaving the original particle with

$$
\left((1-x) P_{0}^{+},-\vec{p}_{\perp}, m_{\perp}^{2} /(1-x) P_{0}^{+}\right)
$$

The intermediate state propagates off-shell by an amount

$$
\Delta p^{-}=m_{\perp}^{2} /(1-x) p_{0}^{+}+m_{1}^{2} / x p_{0}^{+}-m^{2} / p_{0}^{+}
$$

The uncertainty principle in light-cone coordinates then states that the production vertex cannot be localized along the light cone to an accuracy better than 


$$
\Delta x^{+} \sim 2 \hbar / \Delta p^{-} \sim x(1-x) P_{0}^{+} / x^{\prime}
$$

where $k^{\prime}$ " $m_{\perp}^{2} / 2 h$ plays the role of an effective string tension. Equation (3.9) reveals not only the inside-outside picture (1.1) for small $x$ but the outsideinside picture for $x \rightarrow 1$ as first noted in refs. $[10,11]$.

\section{FORMATION DISTRIBUTION IN THE LUND MODEL}

Because of the one-to-one connection [eq. (3.1)] between the constituent formation points $z \frac{ \pm}{n}$ and the momenta $p_{n}^{ \pm}$of the final hadrons, $D(x, z)$ in eqs. (2.7) to $(2.9)$ is completely determined in this model by the fragmentation probability of a quark in momentum space. In the Lund model as in other scaling fragmentation models, the probability that a quark fragments into hadrons carrying light-cone fractions $x_{i}=p_{i}^{+} / p_{0}^{+}$of the initial quark momentum $p_{0}^{+}$is assumed to be given by

$$
F\left(\left\{x_{i}\right\}\right) \prod_{i} d x_{i}=\prod_{i} f\left(u_{i}\right) d u_{i}
$$

where the relative light-cone fractions are

$$
u_{i}=x_{i} /\left(1-\sum_{k=1}^{i-1} x_{k}\right)
$$

and $f(x)$ is a normalized fragmentation function. In the multiflavour standard Lund model $f(x)$ is parametrized as

$$
f(x)=\sum_{q} p_{q}\left(1+C_{q}\right)(1-x)^{C_{q}}
$$

where $\mathrm{p}_{\mathrm{q}}$ is the a priori probability that a given production point involves pair production of a particular flavour $q_{\text {, and }} C_{q}$ are parameters controlling the typical momentum fraction carried away by final hadrons containing that flavour. Inverting eq. (4.2),

$$
x_{i}=u_{i} \prod_{k=1}^{i-1}\left(1-u_{k}\right)
$$

and we see that higher-rank hadrons tend to carry smaller fractional momenta. If we had viewed the generation of the sequence $\left(u_{i}\right)$ according to eq. (4.1) as being timeordered, then we would have erroneously interpreted the fragmentation process as an outside-inside cascade in which lower- $x_{i}$ hadrons are produced on the average after 
higher -x ones. The Lond model avoids this pitfall by associating the $x_{i}$ with the difference of adjacent production points [eqs. (3.1)]:

$$
x_{n}=\left(z_{n-1}^{+}-z_{n}^{+}\right) / 2 L
$$

Solving for the $z_{n}^{+}$using eqs. (4.4) and (4.5) gives

$$
z_{n}^{+}=2 L\left(1-\sum_{k=1}^{n} x_{k}\right)=2 L \prod_{k=1}^{n}\left(1-u_{k}\right)
$$

This shows that, on the average, the production point of higher-rank hadrons with smaller momenta occurs before those of lower rank and higher momenta, in agreement with the inside-outside picture of hadronization. Since $x_{n}$ and $z_{n}^{+}$are completely determined in terms of the first $n$ values of $u_{i}$ ' the density of 'yo-yo' formation points for the $n^{\text {th }}$-rank hadron that carries a momentum fraction $x$ is [14]

$$
D_{n}(x, z)=\int \prod_{k=1}^{n} d u_{k} f\left(u_{k}\right) \delta\left(x-x_{n}\right) \delta\left(z-l_{y n}\right)
$$

where $1_{y n}=\left(z_{n-1}^{+}-z_{n}^{-}\right) / 2$. Integrating over $z$ leads to the $x$ distribution of the $n^{\text {th }}$-rank hadron

$$
D_{n}(x)=\int \prod_{k=1}^{n} d u_{k} f\left(u_{k}\right) \delta\left(x-u_{n} \prod_{i=1}^{n-1}\left(1-u_{i}\right)\right)
$$

Integrating eq. (4.7) over $x$, on the other hand, leads to the density of 'yo-yo' formation points for the $n^{\text {th }}$-rank hadron

$$
\rho_{n}(z)=\int \prod_{k=1}^{n} d u_{k} f\left(u_{k}\right) \delta\left(z-L \prod_{i=1}^{n-1}\left(1-u_{i}\right)+\Delta_{n}\right)
$$

where $\Delta_{n}=z_{n}^{-} / 2=\Sigma^{n} p_{i}^{-} / k$ will be neglected henceforth.

since with $\Delta_{\mathrm{n}} \approx 0$

$$
\delta\left(x-x_{n}\right) \delta\left(z-l_{y_{n}}\right)=\delta\left(x-u_{n} z / L\right) \delta\left(z-L \prod_{k=1}^{n-1}\left(1-u_{k}\right)\right)
$$

we can integrate over $u_{n}$ in eq. (4.7) to express

$$
D_{n}(x, z)=\frac{L}{x} f\left(\frac{x L}{z}\right) \rho_{n}(z)
$$

10 


$$
D_{n}(x)=\int_{x}^{1} \frac{d x^{\prime}}{x^{\prime}} f\left(\frac{x}{x^{\prime}}\right) L \rho_{n}\left(x^{\prime} L\right)
$$

Therefore all distributions of interest are determined by the 'yo-yo' formation densities $\varrho_{n}(z)$. Changing variables to $v_{i}=1-u_{i}$ in eq. (4.9), noting that

$$
\delta\left(z-L \prod_{k=1}^{n} v_{k}\right)=\int d z^{\prime} \delta\left(z^{\prime}-L \prod_{k=1}^{n-1} v_{k}\right) \delta\left(z-v_{n} z^{\prime}\right)
$$

we see that $e_{n}$ for $n>1$ obeys the recursion equation

$$
\rho_{n}(z)=\int_{z}^{L} \frac{d z^{\prime}}{z^{\prime}} f\left(1-z / z^{\prime}\right) \rho_{n-1}\left(z^{\prime}\right)
$$

with $e_{1}(z)=\delta(z-L)$.

Since $f=\left[p_{q} f_{q}\right.$, the distribution for a specific flavour $q$ is obtained simply by replacing $f$ by $p_{q} f_{q}$ in eq. (4.10) and similarly in eq. (4.11). Summing over allrank hadrons gives for the total density of 'yo-yo' formation points of hadrons carrying fractional momentum $x$ and a quark of flavour $q$

$$
D_{Y}(x, z, L)=P_{q} \frac{L}{z} \rho_{r}(z) f_{q}\left(\frac{x L}{z}\right)
$$

Integrating over $z$ leads to the $x$ distribution of final hadrons carrying flavour $q$ :

$$
D(x, L)=p_{q} \int_{x}^{1} \frac{d x^{\prime}}{x^{\prime}} f_{q}\left(\frac{x}{x^{\prime}}\right) L \rho_{Y}\left(x^{\prime} L\right)
$$

where the total density of 'yo-yo' formation points is

$$
\rho_{r}(z)=\sum_{n=1}^{\infty} \rho_{n}(z)=\delta(z-L)+\sum_{n=2}^{\infty} \rho_{n}(z)
$$

In the standard Lond model, where $c_{q}=c$ for all flavours, except for certain rare flavours with $p_{q} \ll 1$ (such as diquarks), direct integration of eq. (4.9) neglecting $\Delta_{n}$ gives

$$
\rho_{n}(z)=\frac{(1+c)^{n-1}}{(n-2) !} \frac{1}{L}\left(\frac{z}{L}\right)^{c}(\ln L / z)^{n-2}, n \geq 2
$$

11 
so that from eq. (4.15)

$$
\rho_{Y}(z)=\delta(z-L)+\frac{1+C}{z}
$$

For more general fragmentation functions, we must solve on account of eq. (4.12) the integral equation

$$
\rho_{Y}(z)=\delta(L-z)+\int_{z}^{L} \frac{d z^{\prime}}{z^{\prime}} f\left(1-\frac{z}{z^{\prime}}\right) \rho_{Y}\left(z^{\prime}\right)
$$

In certain cases eq. (4.17) can be solved efficiently using Mellon transforms [14].

Given eq. (4.16), the 'yo-yo' formation-length distribution in the standard Lung model is therefore

$$
\begin{aligned}
D_{Y}(x, z, L)= & p_{q}\left(1+C_{q}\right) \frac{L}{z}\left(1-\frac{x L}{z}\right)^{C_{q}} \\
& \times\left\{\delta(z-L)+\frac{1+C}{z} \theta(L-z)\right\} \theta(z-x L)
\end{aligned}
$$

with

$$
D(x, L)=p_{q} \frac{1+C}{x}(1-x)^{C q}\left(1+x \frac{C_{q}-C}{1+C}\right)
$$

From eq. (4.19) we see that $C_{q}$ controls the $x \rightarrow 1$ form of the distribution of hadrons containing flavour $q$, while after summing over $q$ and using $\left[p_{q}=1\right.$, we see that $1+\mathrm{C}$ is the total rapidity density of hadronic clusters in the central $\mathrm{x} \rightarrow 0$ region. The final rapidity of pions after decay of heavier clusters is, of course, several times $1+C$. This is why, in the standard Lond model, $C_{q}=c \approx 0.3$ for the abundant flavours $q=u, d$.

Since the formation length of the constituent $q$ is always $x L$ less than the formation length of the 'yo-yo' according to eq. (3.6), the distribution of constituent formation lengths is simply

$$
D_{c}(x, z, L)=D_{r}(x, z+x L, L)
$$

12 
Finally, we note that the average 'yo-yo' formation length computed in ref. [14] is related to $D_{y}$ as

$$
\left\langle\ell_{y}(x)\right\rangle=\int_{0}^{L} d z z D_{Y}(x, z, L) / D(x, L)
$$

To illustrate the characteristic difference between the functional form of $\left\langle 1_{y}(x)\right\rangle$ and $\left\langle l_{C}(x)\right\rangle=\left\langle l_{y}(x)\right\rangle-x L_{1}$, consider first the case $C=0, C_{q}=1$ for simplicity. In this case $D(x)=\left(1-x^{2}\right) / x$ and

$$
\left\langle\ell_{y}(x)\right\rangle=x L\left[\frac{\ln \left(1 / x^{2}\right)}{1-x^{2}}\right]
$$

and

$$
\left\langle\ell_{c}(x)\right\rangle=x L\left[\frac{\ln \left(1 / x^{2}\right)-1+x^{2}}{1-x^{2}}\right]
$$

It is interesting to note that even for $x \rightarrow 0,\left\langle 1_{C}(x)\right\rangle \approx\left\langle 1_{y}(x)\right\rangle \approx x L \ln 1 / x^{2}$ differs from the naive inside-outside cascade picture [in which $l(x)=x I_{\text {] }}$ by an enhancement factor $\ln 1 / \mathrm{x}^{2}$. This factor arises because low-rank hadrons can sometimes carry low momentum while produced at larger distances. Since, in practice, $x \geq\langle m\rangle / E_{i}$, eqs. (4.22) and (4.23) indicate that the formation zone of the slow secondaries increases logarithmically with incident energy, $\left\langle 1\left(\mathrm{x}_{\min }\right)\right\rangle \sim(2\langle\mathrm{~m}\rangle / \mathrm{k}) \ln \left(\mathrm{E}_{\mathrm{i}} /\langle\mathrm{m}\rangle\right)$. This effect may have interesting consequences for ultrarelativistic heavy-ion collisions and lead to a limiting initial energy density $\varepsilon_{0}$ since dN/dy increases as In $E_{i}$ while $\varepsilon_{0} \propto d N / d y\left\langle l_{\min }\right\rangle^{-1}$.

In fig. $3,\left\langle l_{Y}(x)\right\rangle$ and $\left\langle l_{C}(x)\right\rangle$ are plotted for the case $c=0.3, c_{q}=1.3$. The interesting point to note is that $\left\langle l_{C}(x)\right\rangle$ has a maximum around an intermediate $x \approx 0.3$ and thus decreases for both larger and smaller $x !$ As emphasized in connection with eq. (3.9), this feature also emerges from bremsstrahlung models although the position of the maximum is model-dependent. This would imply at first sight that nuclear attenuation should have a minimum for some intermediate value of $x$. However, in the small $\mathrm{x}$ domain, the higher-order terms $\mathrm{do}_{\mathrm{n}}$ in eq. (2.2) will contribute and could compensate for the suppression of $\mathrm{do}_{0}$.

Equations (4.13) and (4.20) show that there are two contributions to $D_{c}$ and $D_{y}$ in general: firstly, there is a delta function fixing $z=L$ for $D_{y}$ and $z=(1-x) L$ 
for $D_{C}$ owing to the first-rank hadron; secondly there is a continuum contribution that peaks at lower $z$ for a fixed $x$ arising from higher-rank hadrons. We note that only the delta-function contribution to $D_{C}$ was considered in refs. [10] and [11], while only the average 'yo-yo' formation point (4.21) was considered in ref. [14].

\section{FRAGMENTATION OF HIGH-ENERGY HADRONS}

The distributions of formation lengths given by eqs. (4.18) and (4.20) apply to fragmentation of elementary strings between current q $\bar{q}$ pairs. Supplemented by eq. (2.8), they can thus best be tested by studying the attenuation of hadrons produced by leptons in nuclei. Unfortunately, the existing lepto-production data are either integrated over a large range of parameters [17] or not accurate enough [18] to allow a meaningful comparison at this time.

Given this situation we focus on recent data on fragmentation of $30 \mathrm{GeV}$ pions into protons and antiprotons [15]. However, the application of the Lund model to hadron-induced reactions requires an extension of the original model [13]. This necessarily involves further model assumptions. In ref. [19] one such extension was proposed. There, the leading hadron loses longitudinal momentum $\mathrm{p}^{+}$and picks up $\mathrm{p}^{-}$ momentum according to a scaling distribution $d \mathrm{p}^{+} \mathrm{dp}^{-} / \mathrm{p}^{+} \mathrm{p}^{-}$in each successive collision in the nucleus. Hadronization via the original Lund model is assumed to take place outside the nucleus: nuclear attenuation arises only because the leading hadron loses $E+p$ and gains $E-p$ in traversing the nucleus. In the context of our formulation (2.2), do in eq. (2.7) would be computed in that model with $\sigma^{*}=\sigma_{h}=\sigma_{i}$. Since this option for $\sigma^{*}$ and $\sigma_{h}$ is a special case of eq. (2.7) and, as we shall see, disagrees with the data, we will consider a more general extension of the Lund model based on ideas of the additive quark model [20] and valon model [21].

We will assume that fragmentation of a high-energy hadron is described by the breaking of a colour string between one of the hadronic constituents and the interaction point. For the process $\pi^{-} \rightarrow \bar{p}$, we thus assume that the $\bar{p}$ is produced from the fragmentation of the $\bar{u}$ "valon" in $\pi^{-}$. We denote by $x_{v}$ the fraction of the (light-cone) momentum of the initial hadron which is carried by this constituent. Then the final hadron carries the $x / x_{v}$ fraction of the constituent momentum. Consequently, the density of final hadrons produced in the fragmentation of that valon at the distance $\Delta z$ from the interaction point is 


$$
\frac{1}{x_{v}} D\left(x / x_{v}, \Delta z, x_{v} L\right)
$$

where $D(x, \Delta z, L)$ is given by eqs. (4.18) and (4.20) for yo-yo and constituent lengths, respectively.

Denoting now the valon momentum distribution in the initial hadron by $F\left(x_{v}\right)$, we obtain for the ratio of production rates on the nucleus and on hydrogen

$$
\begin{aligned}
R_{A}=\int d^{2} b & \int d z \rho(z, b) \int_{z}^{\infty} d z^{\prime} W_{0}\left(z, z^{\prime}\right) \\
& \times\left\{D_{\text {eff }}\left(x, z^{\prime}-z, L\right) / \int_{0}^{\infty} d z^{\prime \prime} D_{\text {eff }}\left(x, z^{\prime \prime}, L\right)\right\}
\end{aligned}
$$

where $W_{0}\left(z, z^{\prime}\right)$ is given by eq. (2.5) and where $D_{\text {eff }}(x, \Delta z, L)$ is the density of final hadrons produced at a distance $\Delta z$ from the interaction point, as given by

$$
\begin{gathered}
\left.\operatorname{Deff}_{\text {eff }} \Delta z, L\right)=\int_{x}^{1} \frac{d x_{v}}{x_{v}} F\left(x_{v}\right) D\left(x / x_{v}, \Delta z, x_{L} L\right) \\
=\frac{1+C q}{L x_{\text {min }}}\left(1-\frac{x}{x_{\text {min }}}\right)^{\frac{C}{q}}\left\{F\left(x_{\text {min }}\right)+\frac{1+c}{x_{\text {min }}} \int_{x_{\text {min }}}^{1} d x_{v} F\left(x_{v}\right)\right\} \\
\theta\left(x_{\text {min }}-x\right) \Theta\left(1-x_{\text {min }}\right)
\end{gathered}
$$

where $x_{\min }=z / L$ for the 'yo-yo' formation length and $x_{\min }=z / L+x$ for the constituent formation length.

To proceed further we need to specify the momentum distribution $F\left(x_{v}\right)$, i.e. to choose a model of fragmentation. We have chosen the valon model [21], which has the advantage of simplicity and of being consistent with data at high energies [20]. For the case of incident $\pi^{-}$, the natural choice [21]

$$
F\left(x_{v}\right)=\theta\left(1-x_{v}\right)
$$

reflects that $\bar{u}$ and $d$ carry on the average only $1 / 2$ the incident energy.

In fig. 4 the function $D_{\text {eff }}(x, \Delta z, L)$ calculated using the constituent length distribution (4.20) is plotted as a function of $\Delta z$ for different values of $x$. One sees that, with increasing $x, D_{\text {eff }}$ is confined to smaller $\Delta z$ regions (outside-inside cascade). The analogous distributions calculated using the 'yo-yo' length are simply shifted to the right by $\mathrm{XL}$ and thus correspond to the inside-outside cascade. 
Using eqs. (5.1) to (5.3) the nuclear attenuation can be calculated, provided one fixes the parameters $C_{1} C_{q}$ and cross-sections $\sigma_{i}, \sigma^{*}$, and $\sigma_{f}$. The parameter $C$ was determined to be equal to $\approx 0.3$ by a Lund model fit to $\mathrm{e}^{+} \mathrm{e}^{-}$data [13] and $\mathrm{c}_{\mathrm{q}}=$ 1.3 for diquarks was chosen to obtain an approximately correct description of the $\bar{p}$ and $\mathrm{p}$ spectra in $\pi$-induced reactions [22]. For $\sigma_{i}$ we used the inelastic, nondiffractive $\pi N$ cross-section calculated assuming that $\sigma_{\text {dif }} \simeq \sigma_{e l}$ :

$$
\sigma_{i}=\sigma_{\text {tot }}-2 \sigma_{e l}
$$

This gives $\sigma_{i} \simeq 17.6 \mathrm{mb}$ for the average of $\pi^{-} \mathrm{p}$ and $\pi^{+} \mathrm{p}$ cross-sections at $30 \mathrm{GeV}$. In the valon model, $a^{\star}$ is the cross-section of the constituent quark. It was calculated from additivity using eq. (5.4) and the corresponding non-diffractive pp crosssection at $30 \mathrm{GeV}(23 \mathrm{mb})$. This gives $\sigma^{*}=\sigma_{q}=7.7 \mathrm{mb}$ and $\sigma^{*}=\sigma_{\bar{q}}=9.9 \mathrm{mb}$ for fragmentation into $p$ and $\bar{p}$, respectively. Finally, $\sigma_{f}$ 's were calculated from protonnucleon and antiproton-nucleon cross-sections using the formula

$$
\sigma_{f}=\sigma_{t o t}-\sigma_{e l}-\frac{1}{2} \sigma_{d i f} \approx \sigma_{t o t}-\frac{3}{2} \sigma_{e l}
$$

because the diffractive breakup of the final hadron does contribute to attenuation. The resulting values of $\sigma_{f}$ at three relevant energies are given in table 1.

Using these cross-sections and the Woods-Saxon nuclear density with the surface parameter $a=0.54 \mathrm{fm}$ and the nuclear radius $r_{A}=1.19 \mathrm{~A}^{1 / 3}-1.61 \mathrm{~A}^{-1 / 3}$, the nuclear attenuation was calculated from eq. (5.1). The results are compared to the data of ref. [15] in fig. 5 .

One sees from figs. $5 a$ and $5 b$ that the valon model describes the data best when the formation length chosen is the constituent length. The 'yo-yo' length gives consistently too little attenuation in the two considered channels. The difference between the choices $l_{C}$ and $l_{y}$ is, however, small at this energy and $x$ value. In figs. $5 c$ and $5 d$ the results of the valon model for $x=0.8$ and $x=0.95$ are shown. One sees that the predicted difference between $1_{C}$ and $l_{y}$ becomes much more significant at these higher values of $x$. Thus further measurements at higher $x$ are needed to clarify which of the formation lengths is in fact relevant for the nuclear attenuation. In figs. $5 \mathrm{a}$ and $5 \mathrm{~b}$ we also plotted nuclear attenuation obtained using the assumption 


$$
\sigma^{*}=\sigma_{f}=\sigma_{i}
$$

This corresponds to the extension of the Lund model proposed in ref. [19], where the final hadrons are assumed to be formed outside the nucleus and the cross-section of the hadronic cluster intersecting in nuclear matter is taken to be that of the initial hadron. One sees from figs. $5 \mathrm{a}$ and $5 \mathrm{~b}$ that this model gives too strong attenuation. Even more importantly, that model predicts no flavour dependence of nuclear attenuation, in contradiction with the data on $\mathrm{p}$ and $\overline{\mathrm{p}}$ production. Higherorder do corrections could remedy the first problem but not the second. We also note that the assumption $\sigma^{*}=\sigma_{f}$, i.e. vanishing formation length, gives much too strong attenuation compared to the data of ref. [15].

We conclude from this exercise that

i) The data of ref. [15] give positive evidence for non-vanishing formation length.

ii) The constituent length seems to describe nuclear attenuation better than the 'yo-yo' length, although the final conclusion must await further data at higher $x$.

iii) The measurements of nuclear attenuation at large $x$ are not only sensitive to the value of the formation length, but they can also make a selection between different models of fragmentation of high-energy hadrons.

We close this section with a comment on large- $x$ data $(x \geq 0.6)$ for the reaction

$$
\pi^{+}+A \rightarrow \eta+X
$$

at $10.5 \mathrm{GeV}$ incident momentum, which were published recently [12]. We found that for $x>0.7$ these data confirm our conclusion: they are consistent with the constituent length with a normal value of the string tension $k=1 \mathrm{GeV} / \mathrm{fm}$, whereas the 'yo-yo' length gives too little attenuation. In ref. [12] a much higher value of $k$ was needed because those authors assumed that $\sigma^{*}=0$ and they neglected fluctuations of the constituent production point. For $x \leq 0.7$ neither 'yo-yo' nor constituent length can describe the very large nuclear attenuation reported in ref. [12]. At these low energies our scaling model assumptions (4.1) must however breakdown, since resonance effects are known to be important there. Data at higher initial energies on (5.7) would help to further test our model. 


\section{CONCLUSIONS AND COMMENTS}

We investigated the formation length of hadrons using the Lund fragmentation model of hadronization and compared the theoretical expectations with the data on the fragmentation of $30 \mathrm{GeV}$ pions into protons and antiprotons. Our conclusions can be summarized as follows.

i) The composite nature of hadrons leads to an ambiguity in the concept of the formation length because different hadronic constituents are, in general, formed at different distances from the interaction point.

ii) We considered two extreme choices of the formation length: the 'yo-yo' length $1_{y}$ corresponding to the point where the two hadronic constituents meet for the first time, and the constituent length $l_{c}$ corresponding to the point where the first hadronic constituent materializes from the vacuum. The distributions of $l_{y}$ and $l_{c}$ were calculated for a given total energy of the system and the energy of the final hadron. The difference between $l_{y}$ and $l_{C}$ is equal to the momentum divided by the string tension. The 'yo-yo' length $1_{y}$ leads to the inside-outside picture of hadronization, while $1_{c}$ leads to an outside-inside picture for large-x ( $(\geq 0.3)$ secondaries.

iii) These results were used to estimate the attenuation of hadrons produced in nuclear matter. Comparison with the data of refs. [12] and [15], although model-dependent, indicates that the constituent length $1_{C}$ seems to describe the observed attenuation better than the 'yo-yo' length $1_{y}$. This somewhat surprising result implies that the newly born high-x hadrons interact rather early in nuclear matter and that their space-time ordering corresponds to the outside-inside cascade.

iv) Since, at a fixed initial energy, the difference between $l_{c}$ and $1_{y}$ increases with increasing $x$, the attenuation of hadrons should increase with increasing $x$ if the constituent length is indeed relevant. Data at $x \geqslant 0.7$ should thus be of great interest for the determination of the hadronic formation length.

v) The specific extension of the Lund model we proposed leads to a definite flavour dependence of nuclear attenuation, which agrees with the smaller attenuation observed for p's compared to $\bar{p}^{\prime} s$. 
In closing we note the following points:

a) As we emphasized in section 5, to test the possibility of an outside-inside cascade of high-x hadronization, we had to extend the original Lund model to hadroninduced processes. Since this extension is not unique and involves several parameters which are difficult to control, the conclusions must be considered as tentative. For a reliable determination of the formation length, high-precision leptoproduction data are necessary. Once the distribution of formation lengths is constrained from lepto-production data, the measurements of the nuclear attenuation in hadron-induced processes could become an important tool in the investigation of the mechanism of hadron fragmentation.

b) The calculations presented here should be improved for more quantitative comparisons with future data. In particular, the higher-order terms do ${ }_{n}$ in eq. (2.2) need to be estimated. For that purpose more information on inelasticity in hadronnucleon interactions needs to be included in the formulation of the model.

c) In view of the results obtained in the present paper it would be of interest to investigate hadron formation lengths in other models of hadronization, as, for example, the parton cascade model [23]. Nuclear attenuation data could provide important constraints on such models. However, meaningful tests of models require as usual comparison with data at the level of $10 \%$.

We wish to thank D. Amati, M. Ciafaloni, F. Halzen, P. Hoyer, R. Petronzio, L. Van Hove and K. Zalewski for useful comments. M.G. acknowledges partial support from the office of High Energy and Nuclear Physics of DOE under contract DE-ACO376 SF00098 and from the CERN Theory Division. 


\section{REFERENCES}

[1] L. Landau and I.Ya. Pomeranchuk, Dokl. Akad. Nauk 22 (1953) 535 and 735.

[2] E.L. Feinberg and I.Ya. Pomeranchuk, Nuovo Cimento Suppl. $\underline{3}$, Ser. 10 (1956) 652.

[3] E.L. Feinberg, Sov. Phys.-JETP 23 (1966) 132.

[4] J.D. Bjorken, in Current-induced interactions, Proc. Int. Summer Institute of Theoretical Physics, Hamburg, 1975, Lecture Notes in Physics (Springer, Berlin, 1976), vol. 56, p. 73; Proc. 1979 SLAC Summer Institute on Particle Physics (ed. A. Mosher) (SLAC Report 224, Stanford, 1980); Proc. 2nd Conf. on Physics in Collision, Stockholm, 1982 (eds. P. Carlson and P. Trower) (Plenum, New York, 1983), p. 343.

[5] M. Miesowick, Acta Phys. Pol. B3 (1972) 105.

[6] 0. Kancheli, JETP Lett. 18 (1973) 274.

[7] K. Gottfried, Proc. 5th Int. Conf. on High Energy Physics and Nuclear Structure, Uppsala, Sweden, June 1983.

K. Gottfried, Phys. Rev. Lett. 32 (1974) 957.

F. Low and F. Gottfried, Phys. Rev. D17 (1978) 2487.

[8] L. Stodolsky, Proc. Oxford Colloquium on Multiparticle Reactions, 1975, p. 577.

[9] N.N. Nikolaev, Z. Phys. C4 (1980) 291 and Usp. Fiz. Nauk 134 (1981) 369.

N.N. Nikolaev, A.Ya. Ostapchuk and V.R. Zoller, preprint CERN-TH.2541 (1979);

N.N. Nikolaev and A.Ya. Ostapchuk, preprint CERN-TH.2575 (1979).

[10] B.Z. Kopelyovich and L.I. Lapidus, Proc. 6th Balaton Conf. on Nuclear Physics, Balatonfüred, 1983, p. 73.

[11] B.Z. Kopelyovich and F. Niedermeyer, Yad. Fiz. 42 (1985) 797 and Dubna preprint E2-834 (1984).

[12] G.S. Bitsadze et al., Nucl. Phys. B279 (1986) 770.

[13] B. Andersson, G. Gustafson, G. Ingelman and T. Sjöstrand, Phys. Rep. 27 (1983) 31.

[14] T. Chmaj, Formation time in the Lund model, Cracow preprint TPJU 86-9 (1986), to be published in Acta Phys. Pol. B.

[15] M.C. Abreu et al., Z. Phys. C25 (1984) 115. 
[16] A. Białas, Acta Phys. Pol. B11 (1980) 475 and B15 (1984) 647; Z. Phys. C26 (1984) 301

A. Białas and T. Chmaj, Phys. Lett. 133B (1983) 241.

[17] L.S. Osborne et al., Phys. Rev. Lett. 40 (1978) 1624.

[18] A. Arvidson et al., Nucl. Phys. B246 (1984) 381.

D.R.O. Morrison, preprint CERN-EP/83-159 (1983) and private communication from T. Coghen.

[19] B. Anderson et al., Nucl. Phys. B281 (1987) 289.

[20] A. Białas and E. Białas, Phys. Rev. D20 (1979) 2854.

A. Dar and F. Takagi, Phys. Rev. Lett. $\underline{44}$ (1980) 768 .

[21] R. Hwa, Phys. Rev. D22 (1980) 759 and 1593.

[22] A.E. Brenner et al., Phys. Rev. D26 (1982) 1497.

[23] A. Bassetto, M. Ciafaloni and G. Marchesini, Phys. Rep. 100 (1983) 201. 
Table 1

Final hadron cross-sections in nuclear matter ${ }^{*}$ )

\begin{tabular}{|c|l|c|c|}
\hline $\begin{array}{l}\text { Energy } \\
(\mathrm{GeV})\end{array}$ & $\mathrm{x}$ & $\begin{array}{c}\sigma_{\mathrm{f}} \text { (proton) } \\
(\mathrm{mb})\end{array}$ & $\begin{array}{c}\sigma_{\mathrm{f}} \text { (antiproton) } \\
(\mathrm{mb})\end{array}$ \\
\hline 15 & 0.5 & 25.8 & 35.9 \\
\hline 24 & 0.8 & 24.2 & 33.9 \\
\hline 28.5 & 0.95 & 23 & 32.1 \\
\hline
\end{tabular}

*) Based on compilations of V. Flaminio et al. reports CERN-HERA 83-01 (1983) and 79-03 (1979). 


\section{Fiqure captions}

Fig. 1 : Nuclear attenuation of a final hadron $h_{f}$ formed at a distance $l$ from the interaction point of the incident hadron $h_{i}$. The intermediate state ${ }^{*}$ is attenuated with cross-section $\sigma^{*}$.

Fig. 2 : Two formation lengths in the Lund model. The 'yo-yo' length $l_{y}$ corresponds to the points $\mathrm{H}_{i}$ where the two hadronic constituents meet for the first time. These lengths obey the inside-outside cascade picture. The constituent length $l_{C}$ corresponds to the point $P_{i}$ where the first constituent of the hadron $\mathrm{H}_{i}$ is created. It leads, in contrast, to an outside-inside cascade picture for large- $x$ hadrons.

Fig. 3 : The average formation length as a function of $x$. The curve labelled 'yo-yo' length corresponds to the result of ref. [14], whereas the curve for the constituent length shows the novel outside-inside aspect of hadronization for $x \geq 0.3$. The simple inside-outside cascade picture corresponds to the dashed line.

Fig. 4 : The distribution of constituent lengths $1_{C}$ in the valon model for different $x$. Note that both large- $x$ and small-x distributions are peaked at small $l_{c^{\prime}}$ reflecting the behaviour shown in fig. 3 .

Fig. 5 : Attenuation of protons and antiprotons produced in nuclear matter by $30 \mathrm{GeV}$ pions in the valon model. Full lines correspond to the constituent lengths. Dashed lines correspond to the 'yo-yo' lengths. The data points are from ref. [15].

a) $\pi^{-} \rightarrow \bar{p}$ at $x=0.5$, b) $\pi^{-} \rightarrow p$ at $\left.x=0.5, c\right) \pi^{-} \rightarrow \bar{p}$ at $x=0.8$ and 0.95 , d) $\pi^{-} \rightarrow \mathrm{p}$ at $\mathrm{x}=0.8$ and 0.95 . The dash-dotted line corresponds to the model of ref. [19] assuming eq. (5.6) with $\mathrm{R}_{\mathrm{A}}$ given by (2.12). The lower hatched line corresponds to vanishing formation length. The upper hatched line corresponds to no attenuation after the first interaction point with $\mathrm{R}_{\mathrm{A}}$ given by (2.11). 


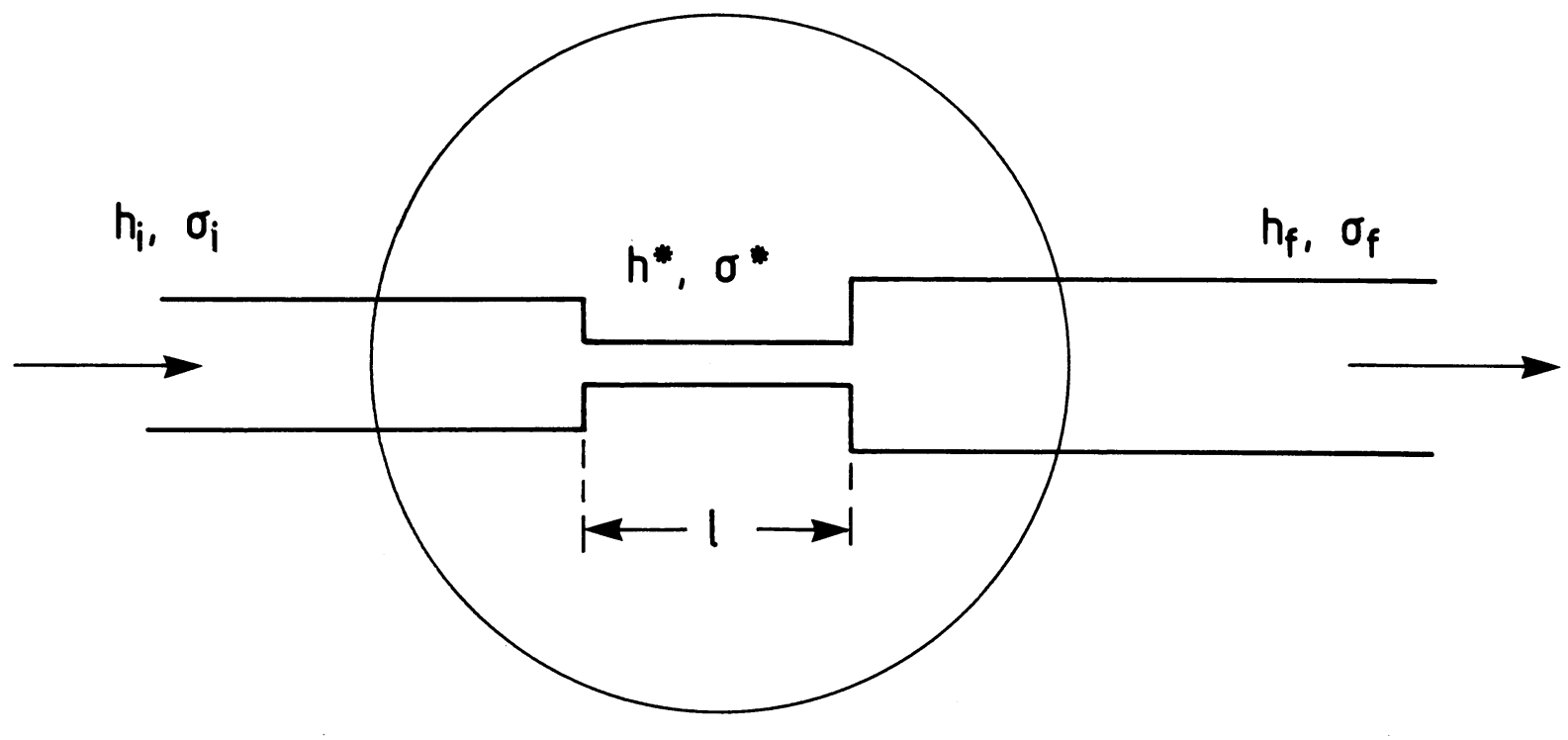

Fig. 1

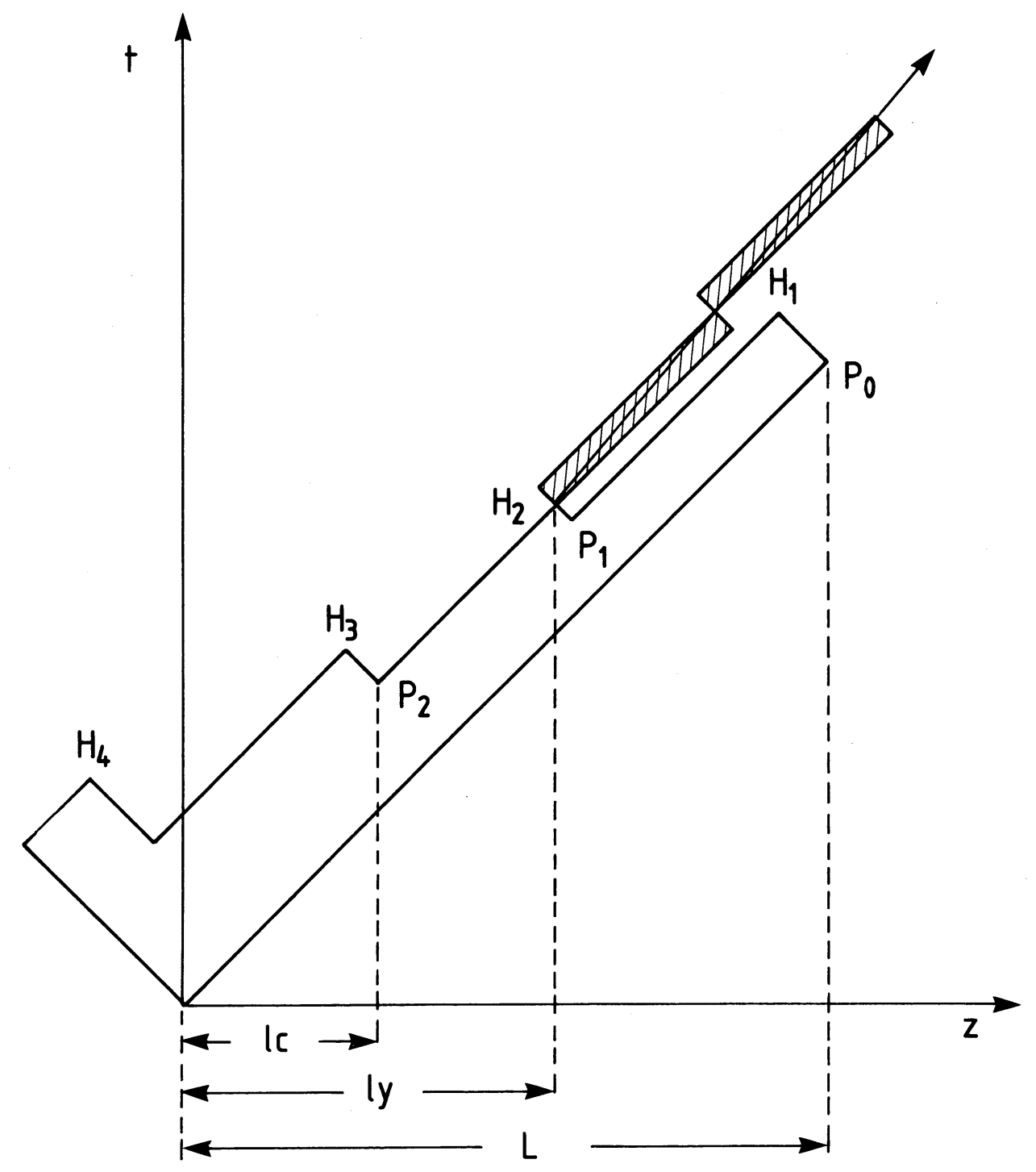

Fig. 2 

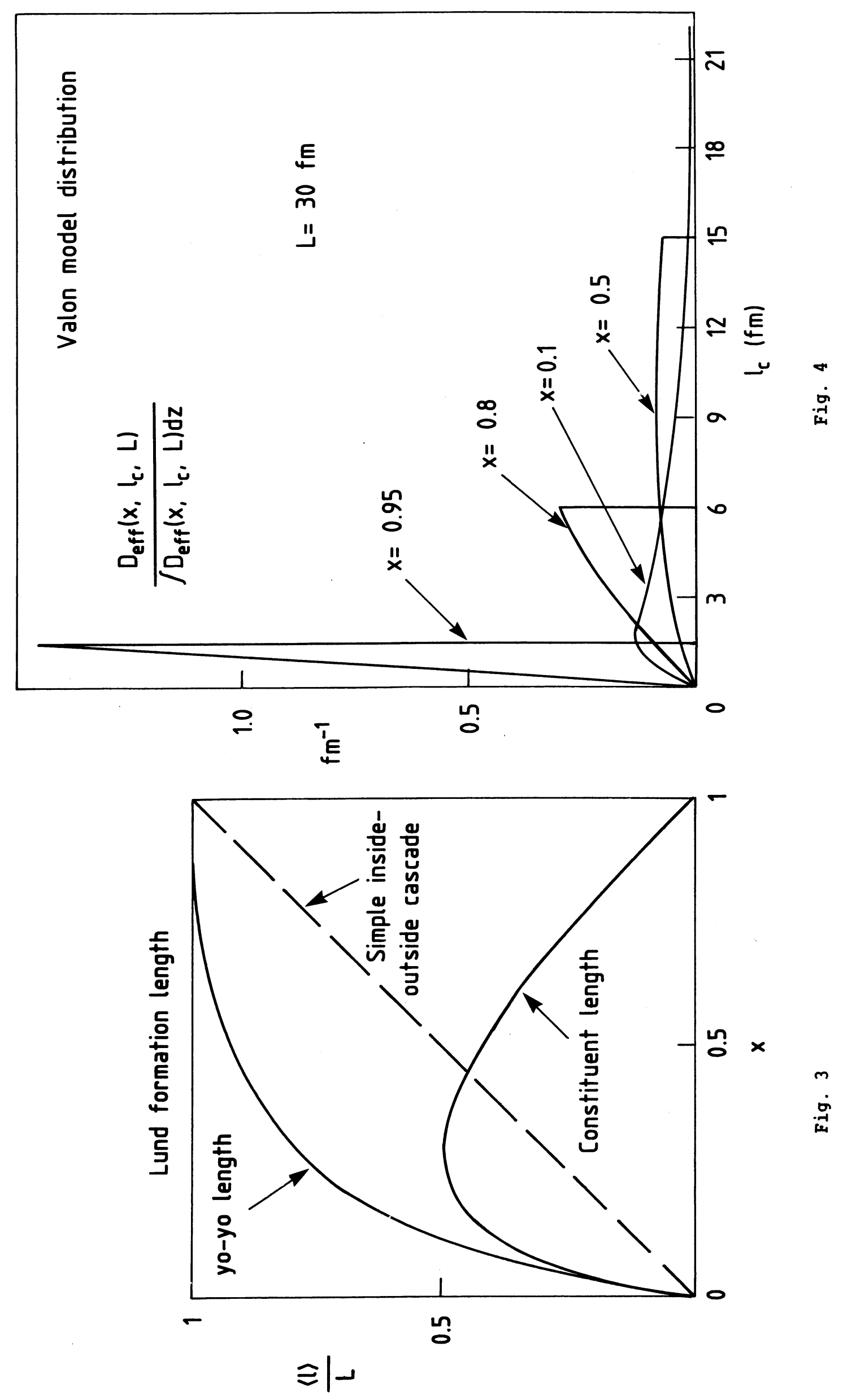


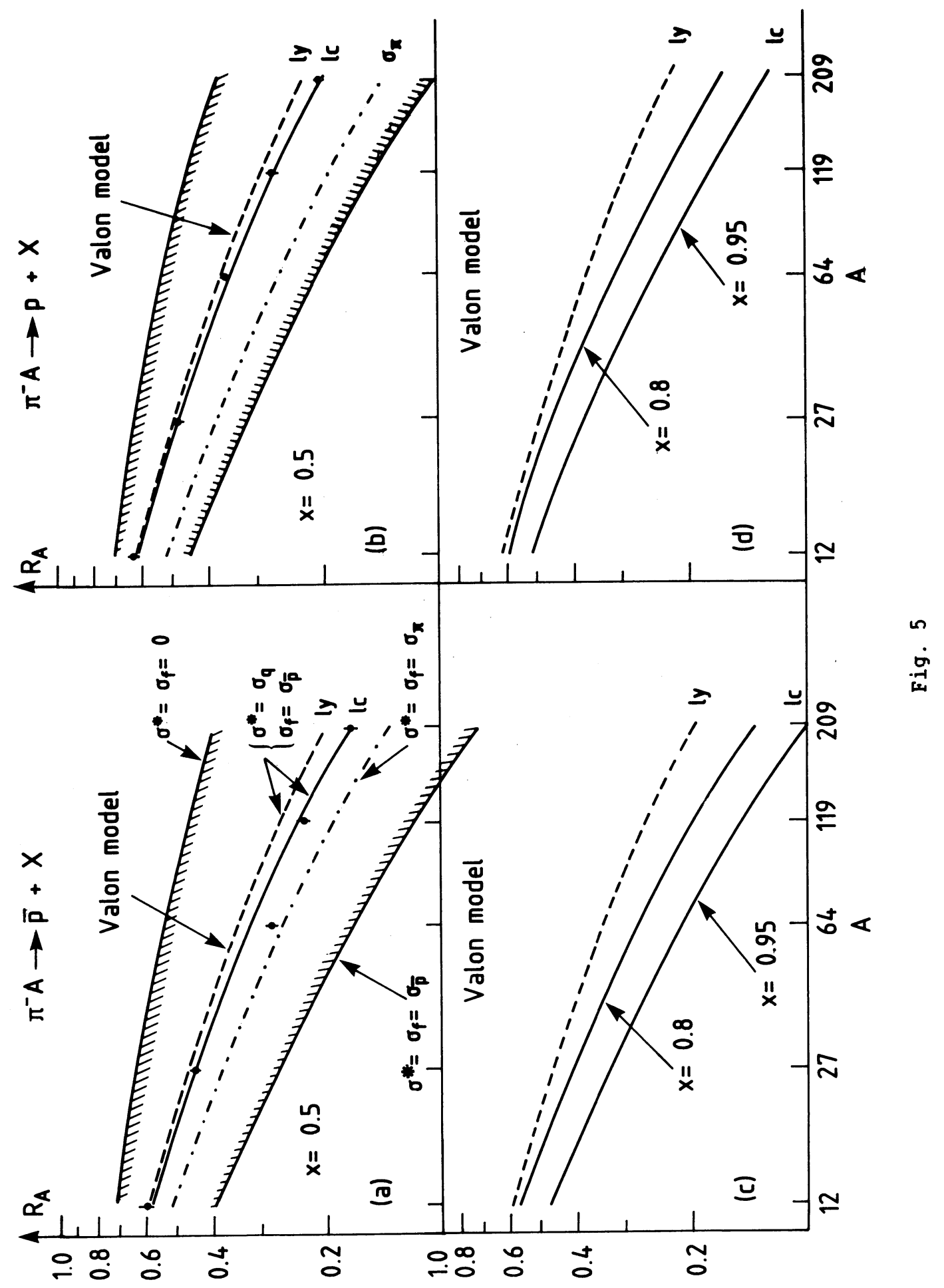

